International Journal of Pure and Applied Mathematics

Volume 90 No. 2 2014, 195-203

ISSN: 1311-8080 (printed version); ISSN: 1314-3395 (on-line version)

url: http://www.ijpam.eu

doi: http://dx.doi.org/10.12732/ijpam.v90i2.9

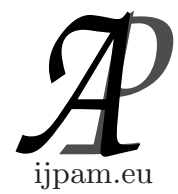

\title{
EXISTENCE OF SOLUTIONS FOR IMPULSIVE QUASILINEAR MIXED VOLTERRA-FREDHOLM TYPE INTEGRODIFFERENTIAL EQUATIONS
}

\author{
F. Paul Samuel ${ }^{1}$, K. Balachandran ${ }^{2}$, T. Gunasekar ${ }^{3}$ \\ ${ }^{1}$ Department of Mathematics and Physics \\ University of Eastern Africa \\ Baraton, Eldoret, 2500-30100, KENYA \\ ${ }^{2}$ Department of Mathematics \\ Bharathiar University \\ Coimbatore, 641 046, INDIA \\ ${ }^{3}$ Department of Mathematics \\ Karunya University \\ Coimbatore, 641 114, INDIA \\ ${ }^{3}$ School of Advanced Sciences \\ Fluid Dynamics Division \\ VIT University \\ Vellore, 632 014, INDIA
}

\begin{abstract}
The paper deals with the study of existence of solutions of quasilinear mixed volterra-Fredholm-type integrodifferential equations with nonlocal and impulsive conditions in Banach spaces. The results are obtained by using a fixed point technique and semigroup theory.
\end{abstract}

AMS Subject Classification: $34 \mathrm{~A} 37,34 \mathrm{G} 60,34 \mathrm{G} 20$

Key Words: semigroup, mild solution, impulsive conditions, evolution equation, fixed point

Received: October 1, 2013

(C) 2014 Academic Publications, Ltd. url: www.acadpubl.eu

${ }^{\S}$ Correspondence author 


\section{Introduction}

Many evolution process are characterized by the fact that at certain moments of time they experience a change of state abruptly. These processes are subject to short-term perturbations whose duration is negligible in comparison with the duration of the process. Consequently, it is natural to assume that these perturbations act instantaneously, that is, in the form of impulses. It is known, for example, that many biological phenomena involving thresholds, bursting rhythm models in medicine and biology, optimal control model in economics, pharmacokinetics and frequency modulated systems, do exhibit impulsive effects. Thus differential equations involving impulsive effects appear as a natural description of observed evolution phenomena of several real world problems.

The impulsive condition is the combination of traditional initial value problem and short-term perturbations whose duration can be negligible in comparison with the duration of process. They have advantages over traditional initial value problem because they can be used to model phenomena that cannot be modelled by traditional initial value problem. Recently, the study of the impulsive differential equations has attracted a great deal of attention. The theory of impulsive differential equations is an important branch of differential equations $[7,10,12]$.

Several authors have studied the existence of solutions of abstract quasilinear evolution equations in Banach space [1, 2, 3, 4, 5, 13]. Bahuguna [1], Oka [8] and Oka and Tanaka [9] discussed the existence of solutions of quasilinear integrodifferential equations in Banach spaces. Kato [6] studied the nonhomogeneous evolution equations where as Chandrasekaran [4] proved the existence of mild solutions of the nonlocal Cauchy problem for a nonlinear integrodifferential equation. The aim of this is to prove the existence and uniqueness of mild solutions of impulsive quasilinear mixed volterra-Fredholm-type integrodifferential equation of the form

$$
\begin{gathered}
u^{\prime}(t)+A(t, u) u(t)=f\left(t, u(t), \int_{0}^{t} g(t, s, u(s)) d s, \int_{0}^{b} k(t, s, u(s)) d s\right), \\
u(0)+h(u)=u_{0} \\
\Delta u\left(t_{i}\right)=I_{i}\left(u\left(t_{i}\right)\right), \quad i=1,2,3, \ldots, m, 0<t_{1}<t_{2}<, \ldots t_{m}<T
\end{gathered}
$$

where $A(t, u)$ be the infinitesimal generator of a $C_{0}$-semigroup in a Banach space $X$. Let $\mathcal{P C}([0, T] ; X)$ consist of functions $u$ from $[0, T]$ into $X$, such that $u(t)$ is continuous at $t \neq t_{i}$ and left continuous at $t=t_{i}$, and the right limit $u\left(t_{i}^{+}\right)$exists for $i=1,2,3, \ldots m$. Evidently $\mathcal{P C}([0, T], X)$ is a Banach space 
with the norm

$$
\|u\|_{\mathcal{P C}}=\sup _{t \in[0, T]}\|u(t)\| .
$$

Let $u_{0} \in X, f:[0, T] \times X \times X \times X \rightarrow X, g: \Omega \times X \rightarrow X, k: \Omega \times X \rightarrow X$, $h: \mathcal{P C}([0, T]: X) \rightarrow X$ and $\Delta u\left(t_{i}\right)=u\left(t_{i}^{+}\right)-u\left(t_{i}^{-}\right)$constitutes an impulsive condition. Here $[0, T]=J$ and $\Omega=\{(t, s): 0 \leq s \leq t \leq T\}$. The results obtained in this paper are generalizations of the results given by Balachandran and Uchiyama [3] and Pazy [11].

\section{Preliminaries}

Let $X$ and $Y$ be two Banach spaces such that $Y$ is densely and continuously embedded in $X$. For any Banach spaces $Z$ the norm of $Z$ is denoted by $\|\cdot\|$ or $\|\cdot\|_{Z}$. The space of all bounded linear operators from $X$ to $Y$ is denoted by $B(X, Y)$ and $B(X, X)$ is written as $B(X)$. We recall some definitions and known facts from Pazy [11].

Definition 2.1. Let $S$ be a linear operator in $X$ and let $Y$ be a subspace of $X$. The operator $\tilde{S}$ defined by $D(\tilde{S})=\{x \in D(S) \cap Y: S x \in Y\}$ and $\tilde{S} x=S x$ for $x \in D(\tilde{S})$ is called the part of $S$ in $Y$.

Definition 2.2. Let $B$ be a subset of $X$ and for every $0 \leq t \leq T$ and $b \in B$, let $A(t, b)$ be the infinitesimal generator of a $C_{0}$ semigroup $S_{t, b}(s), s \geq 0$, on $X$. The family of operators $\{A(t, b)\},(t, b) \in[0, T] \times B$, is stable if there are constants $M \geq 1$ and $\omega$ such that

$$
\begin{gathered}
\rho(A(t, b)) \supset(\omega, \infty) \quad \text { for }(t, b) \in[0, T] \times B, \\
\left\|\prod_{j=1}^{k} R\left(\lambda: A\left(t_{j}, b_{j}\right)\right)\right\| \leq M(\lambda-\omega)^{-k}
\end{gathered}
$$

for $\lambda>\omega$ every finite sequences $0 \leq t_{1} \leq t_{2} \leq \cdots \leq t_{k} \leq T, b_{j} \in B, 1 \leq j \leq k$. The stability of $\{A(t, b)\},(t, b) \in[0, T] \times B$ implies (see [11]) that

$$
\left\|\prod_{j=1}^{k} S_{t_{\mathrm{j}}, b_{\mathrm{j}}}\left(s_{j}\right)\right\| \leq M \exp \left\{\omega \sum_{j=1}^{k} s_{j}\right\}, \quad s_{j} \geq 0
$$

and any finite sequences $0 \leq t_{1} \leq t_{2} \leq \cdots \leq t_{k} \leq T, b_{j} \in B, 1 \leq j \leq k$. $k=1,2, \ldots$ 
Definition 2.3. Let $S_{t, b}(s), s \geq 0$ be the $C_{0}$-semigroup generatated by $A(t, b),(t, b) \in J \times B$. A subspace $Y$ of $X$ is called $A(t, b)$-admissible if $Y$ is invariant subspace of $S_{t, b}(s)$ and the restriction of $S_{t, b}(s)$ to $Y$ is a $C_{0}$-semigroup in $Y$.

Let $B \subset X$ be a subset of $X$ such that for every $(t, b) \in[0, T] \times B, A(t, b)$ is the infinitesimal generator of a $C_{0}$-semigroup $S_{t, b}(s), s \geq 0$ on $X$. We make the following assumptions:

$\left(A_{1}\right)$ The family $\{A(t, b)\},(t, b) \in[0, T] \times B$ is stable.

$\left(A_{2}\right) Y$ is $A(t, b)$-admissible for $(t, b) \in[0, T] \times B$ and the family $\{\tilde{A}(t, b)\},(t, b) \in$ $[0, T] \times B$ of parts $\tilde{A}(t, b)$ of $A(t, b)$ in $Y$, is stable in $Y$.

$\left(A_{3}\right)$ For $(t, b) \in[0, T] \times B, D(A(t, b)) \supset Y, A(t, b)$ is a bounded linear operator from $Y$ to $X$ and $t \rightarrow A(t, b)$ is continuous in the $B(Y, X)$ norm $\|$.$\| for$ every $b \in B$.

$\left(A_{4}\right)$ There is a constant $L>0$ such that

$$
\left\|A\left(t, b_{1}\right)-A\left(t, b_{2}\right)\right\|_{Y \rightarrow X} \leq L\left\|b_{1}-b_{2}\right\|_{X}
$$

holds for every $b_{1}, b_{2} \in B$ and $0 \leq t \leq T$.

Let $B$ be a subset of $X$ and $\{A(t, b)\},(t, b) \in[0, T] \times B$ be a family of operators satisfying the conditions $\left(A_{1}\right)-\left(A_{4}\right)$. If $u \in \mathcal{P C}([0, T]: X)$ has values in $B$ then there is a unique evolution system $U_{u}(t, s), 0 \leq s \leq t \leq T$, in $X$ satisfying, (see [11, Theorem 5.3.1 and Lemma 6.4.2, pp. 135, 201-202]

(i) $\left\|U_{u}(t, s)\right\| \leq M e^{\omega(t-s)}$ for $0 \leq s \leq t \leq T$. where $M$ and $\omega$ are stability constants.

(ii) $\frac{\partial^{+}}{\partial t} U_{u}(t, s) y=A(s, u(s)) U_{u}(t, s) y$ for $y \in Y$, for $0 \leq s \leq t \leq T$.

(iii) $\frac{\partial}{\partial s} U_{u}(t, s) y=-U_{u}(t, s) A(s, u(s)) y$ for $y \in Y$, for $0 \leq s \leq t \leq T$.

Further we assume that

$\left(A_{5}\right)$ For every $u \in \mathcal{P C}([0, T]: X)$ satisfying $u(t) \in B$ for $0 \leq t \leq T$, we have

$$
U_{u}(t, s) Y \subset Y, \quad 0 \leq s \leq t \leq T
$$

and $U_{u}(t, s)$ is strongly continuous in $Y$ for $0 \leq s \leq t \leq T$.

$\left(A_{6}\right)$ Closed bounded convex subsets of $Y$ are closed in $X$. 
$\left(A_{7}\right)$ For every $(t, b) \in J \times B, f(t, b) \in Y$ and $((t, s), b) \in \Omega \times B, g(t, s, b) \in Y$.

$\left(A_{8}\right) h: \mathcal{P C}([0, T]: B) \rightarrow Y$ is Lipschitz continuous in $X$ and bounded in $Y$, that is, there exist constant $H_{1}>0$ and $H_{2}>0$ such that

$\|h(u)-h(v)\|_{Y} \leq H_{1}\|u-v\|_{\mathcal{P C}}, \quad u, v \in \mathcal{P C}([0, T] ; X)$ and $H_{2}=\|h(0)\|$.

For the conditions $\left.\left(A_{9}\right)\right)$ and $\left(A_{1} 0\right)$ let $Z$ be taken as both $X$ and $Y$.

$\left(A_{9}\right) g: \Omega \times Z \rightarrow Z$ is continuous and there exist constants $G_{1}>0$ and $G_{2}>0$ such that

$$
\begin{gathered}
\left.\int_{0}^{t}\|g(t, s, u)-g(t, s, v)\|_{Z} d s \leq G_{1}\|u-v\|_{Z}\right), \quad u, v \in X, \\
G_{2}=\max \left\{\int_{0}^{t}\|g(t, s, 0)\|_{Z} d s:(t, s) \in \Omega\right\} .
\end{gathered}
$$

$\left(A_{10}\right) k: \Omega \times Z \rightarrow Z$ is continuous and there exist constants $K_{1}>0$ and $K_{2}>0$ such that

$$
\begin{gathered}
\left.\int_{0}^{t}\|k(t, s, u)-k(t, s, v)\|_{Z} d s \leq K_{1}\|u-v\|_{Z}\right), \quad u, v \in X, \\
K_{2}=\max \left\{\int_{0}^{t}\|k(t, s, 0)\|_{Z} d s:(t, s) \in \Omega\right\} .
\end{gathered}
$$

$\left(A_{11}\right) f:[0, T] \times Z \times Z \times Z \rightarrow Z$ is continuous and there exist constants $F_{1}>0$ and $F_{2}>0$ such that

$$
\begin{gathered}
\left\|f\left(t, u_{1}, u_{2}, u_{3}\right)-f\left(t, v_{1}, v_{2}, v_{3}\right)\right\| \leq F_{1}\left[\left\|u_{1}-v_{1}\right\|+\left\|u_{2}-v_{2}\right\|+\left\|u_{3}-v_{3}\right\|\right], \\
\text { for } u_{i}, v_{i} \in X, i=1,2,3 . \\
F_{2}=\max _{t \in[0, T]}\|f(t, 0,0,0)\|_{Z} .
\end{gathered}
$$

Let us take $M_{0}=\max \left\{\left\|U_{u}(t, s)\right\|_{B(Z)}, 0 \leq s \leq t \leq T, u \in B\right\}$.

$\left(A_{12}\right) I_{i}: X \rightarrow X$ is continuous and there exist constant $l_{i}>0$, $i=1,2,3, \ldots, m$ such that

$$
\left\|I_{i}(u)-I_{i}(v)\right\| \leq l_{i}\|u-v\|, \quad u, v \in X \text { and } l_{c}=\left\|I_{i}(0)\right\| .
$$


$\left(A_{13}\right)$ There exist a positive constant $r>0$ such that

$$
\begin{aligned}
& M_{0}\left\{\left\|u_{0}\right\|_{Y}+H_{1} r+H_{2}+T\left[F_{1}\left(r\left[1+G_{1}+K_{1}\right]+G_{2}+K_{2}\right)+F_{2}\right]\right. \\
& \left.\quad+\sum_{i=1}^{m}\left(l_{i} r+l_{c}\right)\right\} \leq r
\end{aligned}
$$

and

$$
\begin{aligned}
q= & \left\{\tilde { K } T \left[\left\|u_{0}\right\|_{Y}+H_{1} r+H_{2}+T\left[F_{1}\left(r\left[1+G_{1}+K_{1}\right]+G_{2}+K_{2}+F_{2}\right]\right.\right.\right. \\
& \left.\left.+\sum_{i=1}^{m}\left(l_{i} r+l_{c}\right)\right]+M_{0}\left[H_{1}+T\left(F_{1}+G_{1}+K_{1}\right)+\sum_{i=1}^{m} l_{i}\right]\right\}<1 .
\end{aligned}
$$

Definition 2.4. A function $u \in \mathcal{P C}([0, T]: X)$ is a mild solution of equations (1.1)-(1.3) if it satisfies

$$
\begin{aligned}
u(t) & =U_{u}(t, 0) u_{0}-U_{u}(t, 0) h(u) \\
& \left.+\int_{0}^{t} U_{u}(t, s)\left[f(s, u(s)), \int_{0}^{s} g(s, \tau, u(\tau)) d \tau, \int_{0}^{b} k(s, \tau, u(\tau)) d \tau\right)\right] d s \\
& +\sum_{0<t_{i}<t} U_{u}\left(t, t_{i}\right) I_{i}\left(u\left(t_{i}\right)\right), \quad 0 \leq t \leq T
\end{aligned}
$$

Definition 2.5. A function $u \in \mathcal{P C}([0, T]: X)$ such that $u(t) \in D(A(t, u(t))$ for $t \in(0, T], u \in C^{1}\left((0, T] \backslash\left\{t_{1}, t_{2}, \ldots, t_{m}\right\}: X\right)$ and satisfies (1.1)-(1.3) in $\mathrm{X}$ is called a classical solution of (1.1)-(1.3) on $[0, T]$,

Further there exists a constant $\tilde{K}>0$ such that for every $u, v \in \mathcal{P C}([0, T]$ : $X$ ) and every $y \in Y$ we have

$$
\left\|U_{u}(t, s) y-U_{v}(t, s) y\right\| \leq \tilde{K} T\|y\|_{Y}\|u-v\|_{\mathcal{P C}} .
$$

\section{Existence Result}

Theorem 3.1. Let $u_{0} \in Y$ and let $B=\left\{u \in X:\|u\|_{X} \leq r\right\}, r>0$. If the assumptions $\left(A_{1}\right)-\left(A_{13}\right)$ are satisfied, then (1.1)-(1.3) has a unique mild solution $u \in \mathcal{P C}([0, T]: Y)$.

Proof. Let $S$ be a nonempty closed subset of $\mathcal{P C}([0, T]: X)$ defined by

$$
S=\left\{u: u \in \mathcal{P C}([0, T]: X),\|u(t)\|_{\mathcal{P C}} \leq r \text { for } 0 \leq t \leq T\right\} .
$$


Consider a mapping $\Phi$ on $S$ defined by

$$
\begin{aligned}
(\Phi u)(t) & =U_{u}(t, 0) u_{0}-U_{u}(t, 0) h(u) \\
& \left.+\int_{0}^{t} U_{u}(t, s)\left[f(s, u(s)), \int_{0}^{s} g(s, \tau, u(\tau)) d \tau, \int_{0}^{b} k(s, \tau, u(\tau)) d \tau\right)\right] d s \\
& +\sum_{0<t_{\mathrm{i}}<t} U_{u}\left(t, t_{i}\right) I_{i}\left(u\left(t_{i}\right)\right) .
\end{aligned}
$$

We claim that $\Phi$ maps $S$ into $S$. For $u \in S$, we have

$$
\begin{aligned}
& \|\Phi u(t)\|_{Y} \leq\left\|U_{u}(t, 0) u_{0}\right\|+\left\|U_{u}(t, 0) h(u)\right\| \\
& +\int_{0}^{t}\left\|U_{u}(t, s)\right\|\left[f(s, u(s)), \int_{0}^{s} g(s, \tau, u(\tau)) d \tau, \int_{0}^{b} k(s, \tau, u(\tau)) d \tau\right) \\
& \quad-f(s, 0,0,0) \| \\
& \quad+\|f(s, 0,0,0)\|] d s+\sum_{0<t_{\mathrm{i}}<t}\left\|U_{u}\left(t, t_{i}\right)\right\|\left[\left\|I_{i}\left(u\left(t_{i}\right)\right)-I_{i}(0)\right\|+\left\|I_{i}(0)\right\|\right] \\
& \leq M_{0}\left\|u_{0}\right\|+M_{0}[H\|u\|+h(0) \| \\
& \quad+M_{0} T\left[F_{1}\left(\|u\|+\left\|\int_{0}^{s} g(s, \tau, u(\tau)) d \tau\right\|+\left\|\int_{0}^{b} k(s, \tau, u(\tau)) d \tau\right\|\right)+F_{2}\right] \\
& \quad+M_{0}\left(l_{i}\|u\|+\left\|I_{i}(0)\right\|\right) \\
& \leq M_{0}\left\{\left\|u_{0}\right\|_{Y}+H_{1} r+H_{2}+T\left[F_{1}\left(r\left[1+G_{1}+K_{1}\right]+G_{2}+K_{2}\right)+F_{2}\right]\right. \\
& \left.\quad+\sum_{i=1}^{m}\left(l_{i} r+l_{c}\right)\right\} .
\end{aligned}
$$

From assumption $\left(A_{13}\right)$, one gets $\|\Phi u(t)\|_{Y} \leq r$. Therefore $\Phi$ maps $S$ into itself.

Moreover, if $u, v \in S$, then

$$
\begin{aligned}
& \|\Phi u(t)-\Phi v(t)\| \leq\left\|U_{u}(t, 0) u_{0}-U_{v}(t, 0) u_{0}\right\|+\left\|U_{u}(t, 0) h(u)-U_{v}(t, 0) h(v)\right\| \\
& \left.+\int_{0}^{t} \| U_{u}(t, s)\left[f(s, u(s)), \int_{0}^{s} g(s, \tau, u(\tau)) d \tau, \int_{0}^{b} k(s, \tau, u(\tau)) d \tau\right)\right] \\
& \left.\quad-U_{v}(t, s)\left[f(s, v(s)), \int_{0}^{s} g(s, \tau, v(\tau)) d \tau, \int_{0}^{b} k(s, \tau, v(\tau)) d \tau\right)\right] \| d s \\
& \quad+\sum_{0<t_{\mathrm{i}}<t}\left\|U_{u}\left(t, t_{i}\right) I_{i}\left(u\left(t_{i}\right)\right)-U_{v}\left(t, t_{i}\right) I_{i}\left(v\left(t_{i}\right)\right)\right\| .
\end{aligned}
$$


Using assumptions $\left(A_{8}\right)-\left(A_{13}\right)$, one can get

$$
\begin{aligned}
\leq & \left\{\tilde { K } T \left[\left\|u_{0}\right\|_{Y}+H_{1} r+H_{2}+T\left[F_{1}\left(r\left[1+G_{1}+K_{1}\right]+G_{2}+K_{2}+F_{2}\right]\right.\right.\right. \\
& \left.\left.+\sum_{i=1}^{m}\left(l_{i} r+l_{c}\right)\right]+M_{0}\left[H_{1}+T\left(F_{1}+G_{1}+K_{1}\right)+\sum_{i=1}^{m} l_{i}\right]\right\}\|u-v\|_{\mathcal{P C}} \\
= & q\|u-v\|_{P C}, \quad u, v \in \mathcal{P C}([0, T] ; X)
\end{aligned}
$$

where $0<q<1$. From this inequality it follows that for any $t \in[0, T]$,

$$
\|\Phi u(t)-\Phi v(t)\| \leq q\|u-v\|_{\mathcal{P C}},
$$

so that $\Phi$ is a contraction on $S$. From the contraction mapping theorem it follows that $\Phi$ has a unique fixed point $u \in S$ which is the mild solution of (1.1)-(1.3) on $[0, T]$. Note that $u(t)$ is in $\mathcal{P C}([0, T]: Y)$ by $\left(A_{6}\right)$ see [11, pp. $135,201-202$ lemma 7.4]. In fact, $u(t)$ is weakly continuous as a $Y$-valued function. This implies that $u(t)$ is separably valued in $Y$, hence it is strongly measurable. Then $\|u(t)\|_{\mathcal{P C}}$ is bounded and measurable function in $t$. Using the relation $u(t)=\Phi u(t)$, we conclude that $u(t)$ is in $\mathcal{P C}([0, T]: Y)$.

\section{Conclusions}

In this paper, we have studied the existence of solutions of quasilinear mixed volterra-type integrodifferential equations with nonlocal and impulsive conditions in Banach spaces. Through semigroup theory and Banach fixed point principle, we have investigated the sufficient conditions for the existence of the system considered.

\section{References}

[1] D. Bahuguna, Quasilinear integrodifferential in Banach spaces, Nonlinear Anal. 24 (1995), 175-183.

[2] K. Balachandran and F. Paul Samuel, Existence of mild solutions for quasilinear integrodifferential equations with impulsive conditions, Electron. J. Diff. Eqns., 2009(2009) No.84, 1-9. 
[3] K. Balachandran and K. Uchiyama, Existence of solutions of quasilinear integrodifferential equations with nonlocal condition, Tokyo. J. Math., 23(2000), 203-210.

[4] M. Chandrasekaran, Nonlocal Cauchy problem for quasilinear integrodifferential equations in Banach spaces, Electron. J. Diff. Eqns., 2007(2007) No. 33, 1-6.

[5] Q. Dong, G. Li and J. Zhang, Quasilinear nonlocal integrodifferential equations in Banach spaces, Electron. J. Diff. Eqns., 2008(2008) No. 19, 1-8.

[6] S. Kato, Nonhomogeneous quasilinear evolution equations in Banach spaces, Nonlinear Anal. 9 (1985), 1061-1071.

[7] V. Lakshmikantham, D. D. Bainov and P. S. Simeonov, Theory of Impulsive differential Equations, World Scientific, Singapore, 1989.

[8] H. Oka, Abstract quasilinear Volterra integrodifferential equations, Nonlinear Anal., 28 (1997), 1019-1045.

[9] H. Oka and N. Tanaka, Abstract quasilinear integrodifferential equtions of hyperbolic type, Nonlinear Anal., 29 (1997), 903-925.

[10] J.Y.Park, K.Balachandran and N.Annapoorani, Existence results for impulsive neutral functional integrodifferential equations with infinite delay, Nonlinear Anal., 71 (2009), 3152-3162.

[11] A. Pazy, Semigroups of Linear Operators and Applications to Partial Differential Equations, Springer, New York, 1983.

[12] A. M. Samoilenko and N. A. Perestyuk, Impulsive Differential Equations, World Scientific, Singapore, 1995.

[13] N. Sanekata, Abstract quasilinear equations of evolution in nonreflexive Banach spaces, Hiroshima Mathematical Journal, 19 (1989), 109-139. 
